\title{
Elucidating the causes and examining the latest clinical findings in pulmonary fibrosis: recent progress and future objectives
}

\section{R.M. du Bois* and T.E. King Jr ${ }^{\#}$}

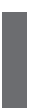
n the last decade, we have witnessed many important advances in the current understanding of the clinical disease process that is now defined as idiopathic pulmonary fibrosis (IPF) and how it differs from the other "members" of the idiopathic interstitial pneumonias (IIPs). We have gained insight into the genetic associations with IPF and new insights into pathogenesis. Importantly, we have begun to distinguish IPF from the other IIPs with much more precision and confidence. Recent investigations have been published that identify clues to potential future therapeutic strategies. Each of these developments has been reflected within the articles in the present issue of European Respiratory Review, providing a comprehensive update across the field of IPF but, most importantly, highlighting areas of more rapid evolution and future potential.

The articles have summarised what are perceived to be the areas of growth in this group of fibrosing lung diseases, and, more specifically, in IPF. The histopathological patterns of fibrosis observed in each of the IIPs are different, and this is also reflected by the clinical behaviour of the individual diseases. Idiopathic usual interstitial pneumonia (UIP) generally progresses in a more aggressive fashion than the other IIPs and each has some distinctive clinical features that distinguish them. Despite these differences, obtaining a consistent and uniform diagnosis of each of the IIPs can be difficult, and further investigation is needed to reduce the inconsistencies in diagnosis between expert and community clinicians. Issues of overlapping patterns of disease and combinations of patterns will be challenges for the future, particularly with regard to concepts of pathogenesis, outcome and treatment.

Despite these confounders, the imaging of patients using high-resolution computed tomography (HRCT) has, in general, enabled a growing number of physicians to make a confident clinical diagnosis without recourse to surgical lung biopsy (SLB). In this regard, confidence in the HRCT pattern can be used to differentiate patients with UIP/IPF from those with other diseases, particularly nonspecific interstitial pneumonia. An important area of future research will be to understand why individuals with an atypical computed tomography (CT) pattern, but who have IPF defined by SLB, appear to have different disease behaviour than those with so-called "classic" disease on CT presentation.

In this context, median survival is observed to be lower among patients with HRCT-defined UIP (i.e. patients whose CT scans are regarded to be typical of UIP with high confidence) than those with a histological diagnosis of UIP but an atypical HRCT [1]. Such potential differences in prognosis between subgroups have important implications for patient management, and particularly for the design of clinical trials. It is significant that current guidelines on the classification of the IIPs emphasise that intervention trials should be discouraged until a concerted effort has been made to establish a confident diagnosis based on currently recognised criteria [2]. Therefore, to maximise the likelihood of achieving positive outcomes in future prospective interventional studies in patients with IPF, efforts must be made to identify and examine prospectively distinct patient subgroups. We must also continue to explore the associations between pace of decline in lung function with specific HRCT patterns, and the role of molecular markers as surrogates for pulmonary function test decline and mortality.

Progress has also been made in understanding the genetic predisposition to IPF and the concept of aberrant wound healing but there are a number of key questions about this disease that still have to be answered. These include: what triggers the epithelial cell injury, especially the injury that is associated with acute exacerbation; why is the disease localised to the periphery of the lung (and the periphery of the secondary pulmonary lobule); how do the fibroblastic foci emerge; and what becomes of them, and why

\section{AFFILIATIONS}

*Dept of Medicine, National Jewish Health, Denver, CO, and \#Dept of Medicine, University of California San Francisco, San Francisco, CA, USA.

\section{CORRESPONDENCE}

R.M. du Bois

Dept of Medicine

National Jewish Health

1400 Jackson Street

Denver

CO 80206

USA

Fax: 13033981452

E-mail: duBoisR@njc.org

\section{STATEMENT OF INTEREST}

R.M. du Bois has received travel grants and is/has been a paid consultant, Steering Committee member and/or Co-Chair of a Steering Committee in the past 5 yrs for Intermune, Actelion, Centocor, Boehringer Ingelheim, Novartis, Genzyme and MondoBiotech. T.E. King Jr has served on advisory boards for Actelion, InterMune, GlaxoSmithKline and ImmuneWorks, and has also served as a consultant for Nektar, Alexza, AstraZeneca, Biogen, Centocor, Fibrogen, Genzyme, Human Genome Sciences, Merck and CoTherix.

European Respiratory Review Print ISSN 0905-9180 Online ISSN 1600-0617 
fibrosis progresses rather than regresses once the "wound" has healed. The importance of the vasculature, including angiogenesis/angiostasis, in the underlying disease process in IPF and, particularly, the influence of the endothelium is still unclear and requires further investigation.

Having realistic expectations for the outcomes of trials in IPF is also important. For example, it appears clear that the honeycombing observed in IPF patients will probably never decrease in extent. Therefore, there is no logic in using this as an end-point in a trial for an investigational therapy. Similar arguments can be proposed for expectations of the extent of lung function improvement that is likely to occur when there is predominant honeycombing present. In this context, it may be too easy to reject a therapy as being ineffective because large lung function improvements are not observed after a trial of investigational therapy. Instead, the focus should be on the recognition of early and less destructive patterns of disease and on intervening when it may be possible to achieve some improvement or, at worst, prevent disease progression.

These arguments touch on the importance of the choice of endpoints in IPF treatment trials. End-points that might be considered as potential primary or secondary outcome measures should be those that might be reasonably expected to reflect an effect of novel drug therapy and include serial measurements of lung function, especially forced vital capacity (FVC), rates of hospital admissions, mortality or a combined end-point of "progression-free survival" using FVC and death. We still do not have well validated biomarkers that can be used as surrogates for functional change and improved survival. We need a validated tool that provides highly reliable and consistent information about the potential course of a patient with IPF. This tool should reliably predict future changes reflective of the disease course over shorter periods of follow-up (e.g. 6-9 months). This would enhance the pace and progress in the clinical trials process by saving money and also improving the rate at which novel agents can be evaluated.

As we continue to debate which outcome measures are the most suitable for IPF trials, progress has been made in identifying potential new therapeutic targets. If we are indeed correct in the belief that IPF is the consequence of repeated cycles of alveolar epithelial cell injury leading to the development of fibroblastic foci, honeycombing and aberrant wound healing, then it is important to identify the triggers for epithelial cell injury and find ways of protecting alveolar epithelial cells from repeated damage. It is also incumbent on us to determine why fibrosis, and even the fibroblastic foci, appears to evolve in a quasimalignant fashion after epithelial cell injury. The role the vasculature plays in the repair process needs to be considered. For example, it is known that even when endothelial cells adjacent to injured epithelial cells appear normal under a light microscope, they show evidence of ultrastructural injury. This suggests that the vasculature (both the alveolar epithelial cell and alveolar capillary wall) may be important in IPF pathogenesis and is not merely a bystander. It even raises the possibility that the endovascular compartment might be the site of the primary injury and that the epithelial cell injury is secondary.

The coagulation cascade represents another potential therapeutic target for the treatment of IPF. Several approaches to targeting the coagulation cascade have been investigated in experimental models of pulmonary fibrosis, including direct thrombin inhibition [3], tissue factor pathway inhibition [4], intratracheal administration of activated protein $C$ [5] and aerosolised heparin [6]. All of these approaches have been observed to reduce the accumulation of collagen in the lungs and the subsequent development of fibrosis. In this regard, a recent study from Japan [7] has shown that treatment with anticoagulation at the time of hospital admission for worsening IPF results in a better outcome following discharge from hospital compared with patients who did not receive anticoagulation treatment.

Other strategies under consideration for treating IPF include antagonism of proteinase activated receptors and a "therapeutic" manipulation of apoptosis. For example, hepatocyte growth factor, a ligand for a receptor tyrosine kinase, exerts an anti-apoptotic effect on epithelial cells but an apoptotic effect on myofibroblasts [8]. Angiotensin receptor blockade represents another possible therapeutic approach to selective apoptosis and derives from evidence that angiotensin II is an important mediator of apoptosis of alveolar epithelial cells [9] and other pro-fibrogenic actions [10]. Experimental studies have shown that administration of an angiotensin- 1 receptor antagonist can attenuate pulmonary fibrosis in vivo [11], while also inhibiting Fas-induced apoptosis in cultured human lung cells [12].

The potent pro-fibrotic, pro-inflammatory vasoconstrictor endothelin- 1 is another potential and logical therapeutic target for IPF, and one for which we already have some clinical data from the large randomised, placebo-controlled Bosentan Use in Interstitial Lung Disease (BUILD)-1 trial. Although the trial did not demonstrate a treatment effect on the primary end-point of change from baseline in 6-min walk distance (6MWD), a trend to delayed time-to-death or disease progression in favour of bosentan was observed in secondary analyses. Post hoc analysis also showed that this trend was more pronounced in the subset of patients with biopsy-proven IPF who had undergone SLB because of atypical HRCT at baseline [13]. These observations will be investigated further in the ongoing, larger BUILD-3 morbidity/mortality study in patients with IPF.

The most ideal target for therapy is to prevent disease initiation. Ideally, this requires: the identification of genetic predisposing factors; screening strategies for genetic susceptibilities being made available to the public; and therapeutically targeting the consequences of the functional abnormality, which result from the genetic anomalies. Cohort studies have identified mutations in surfactant protein $C$ as a rare cause of familial IPF, while mutations in the telomerase genes hTERT and TERC may account for as many as $10 \%$ of cases of familial IPF [14, 15]. Interestingly, mutations in both genes have potential implication for type II alveolar epithelial cell viability; misfolded protein from aberrant surfactant protein $\mathrm{C}$ mutations can block the Golgi apparatus and trigger endoplasmic reticulum stress and apoptosis, and telomerase gene mutations result in shortened telomeres that also trigger apoptosis. However, studies into the importance of genetic predisposition in nonfamilial IPF are less advanced. Although gene expression studies in IPF have identified a number of gene targets, the relative contributions of individual gene variants is unknown. 
Furthermore, the relative importance of genetic versus environmental factors in influencing the development of nonfamilial $\mathrm{IPF}$, and likely familial disease, is also unknown. In the future, by incorporating information from microarrays, genomics and proteomics we may be able to "fingerprint" the disease more precisely, potentially facilitating diagnosis of IPF, identifying the key processes that allow the disease to progress and predicting long-term outcome and the best treatment.

At present, idiopathic pulmonary fibrosis remains a devastating, life-limiting disease of unknown aetiology with an outcome that is worse than many cancers and for which treatment options are limited. The articles in the present issue of the European Respiratory Review shed important new light on many aspects of this disease from bench to bedside. Continued research and investment remain crucial for yielding greater insights into the underlying pathological mechanisms, and how we might exploit this new knowledge for therapeutic gain. By continuing to elucidate the causes and examine the latest clinical findings in idiopathic pulmonary fibrosis, we are moving closer to achieving improvements in patients' lives.

\section{ACKNOWLEDGEMENTS}

The authors would like to thank A. Gray for his role in the literature review and preparation of this manuscript.

\section{REFERENCES}

1 Flaherty KR, King TE Jr, Raghu G, et al. Idiopathic interstitial pneumonia: what is the effect of a multidisciplinary approach to diagnosis? Am J Respir Crit Care Med 2004; 170: 904-910.

2 American Thoracic Society, European Respiratory Society. American Thoracic Society/European Respiratory Society International Multidisciplinary Consensus Classification of the Idiopathic Interstitial Pneumonias. Am J Respir Crit Care Med 2002; 165: 277-304.

3 Howell DC, Goldsack NR, Marshall RP, et al. Direct thrombin inhibition reduces lung collagen, accumulation, and connective tissue growth factor mRNA levels in bleomycin-induced pulmonary fibrosis. Am J Pathol 2001; 159: 1383-1395.

4 Kijiyama N, Ueno H, Sugimoto I, et al. Intratracheal gene transfer of tissue factor pathway inhibitor attenuates pulmonary fibrosis. Biochem Biophys Res Commun 2006; 339: 1113-1119.

5 Yasui H, Gabazza EC, Tamaki S, et al. Intratracheal administration of activated protein $\mathrm{C}$ inhibits bleomycininduced lung fibrosis in the mouse. Am J Respir Crit Care Med 2001; 163: 1660-1668.

6 Günther A, Lübke N, Ermert M, et al. Prevention of bleomycin-induced lung fibrosis by aerosolization of heparin or urokinase in rabbits. Am J Respir Crit Care Med 2003; 168: 1358-1365.

7 Kubo H, Nakayama K, Yanai M, et al. Anticoagulant therapy for idiopathic pulmonary fibrosis. Chest 2005; 128: 1475-1482.

8 Kim WH, Matsumoto K, Bessho K, Nakamura T. Growth inhibition and apoptosis in liver myofibroblasts promoted by hepatocyte growth factor leads to resolution from liver cirrhosis. Am J Pathol 2005; 166: 1017-1028.

9 Li X, Rayford H, Uhal BD. Essential roles for angiotensin receptor AT1a in bleomycin-induced apoptosis and lung fibrosis in mice. Am J Pathol 2003; 163: 2523-2530.

10 Marshall RP, Gohlke P, Chambers RC, et al. Angiotensin II and the fibroproliferative response to acute lung injury. Am J Physiol Lung Cell Mol Physiol 2004; 286: L156-L164.

11 Molteni A, Wolfe LF, Ward WF, et al. Effect of an angiotensin II receptor blocker and two angiotensin converting enzyme inhibitors on transforming growth factor- $\beta$ (TGF- $\beta$ ) and $\alpha$-actomyosin ( $\alpha$ SMA), important mediators of radiation-induced pneumopathy and lung fibrosis. Curr Pharm Des 2007; 13: 1307-1316.

12 Uhal BD, Gidea C, Bargout R, et al. Captopril inhibits apoptosis in human lung epithelial cells: a potential antifibrotic mechanism. Am J Physiol 1998; 275: L1013-L1017.

13 King TE Jr, Behr J, Brown KK, du Bois R, Raghu G. A randomized placebo-controlled trial of bosentan in patients with idiopathic pulmonary fibrosis. Am J Respir Crit Care Med 2007; 176: 1-7.

14 Nogee LM, Dunbar AE 3rd, Wert SE, Askin F, Hamvas A, Whitsett JA. A mutation in the surfactant protein $C$ gene associated with familial interstitial lung disease. $N$ Engl $J$ Med 2001; 344: 573-579.

15 Armanios M, Chen JL, Chang YP, et al. Haploinsufficiency of telomerase reverse transcriptase leads to anticipation in autosomal dominant dyskeratosis congenita. Proc Natl Acad Sci USA 2005; 102: 15960-15964. 